\title{
VINCULACIÓN EN MATERIA DE INNOVACIÓN ENTRE LA UNIVERSIDAD DE GUADALAJARA Y EL SECTOR PRODUCTIVO FARMACÉUTICO EN JALISCO ¿Refleja el tránsito hacia sociedades del conocimiento?
}

\author{
Claudia Fabiola Sánchez Cervantes, Ricardo Arechavala Vargas \\ Universidad de Guadalajara, México
}

\section{PALABRAS CLAVE}

Vinculación

Innovación

Desarrollo económico

Universidad-Industria

Producción

del conocimiento

\section{RESUMEN}

Este trabajo ofrece una caracterización del proceso de vinculación que se lleva a cabo entre la Universidad de Guadalajana (UdeG) y el Sector Productivo Farmacéutico de Jalisco (SFPJ) hacia el llamado modo 2 de Gibbons. Mediante la aplicación de entrevistas no estructuradas a los responsables de innovación en las empresas y a miembros de la universidad y con una revisión documental se muestra: el crecimiento de personas calificadas en las áreas de conocimiento específicas y la inversión gubernamental en el desarrollo de ciencia e innovación, para una temporalidad que comprende del año 2010 al 2015.

\section{KEY WORDS}

Collaborative Work

Industry

University

Innovation

Economic Development

\section{ABSTRACT}

This paper provides a characterization about the collaborative work between Universidad de Guadalajara and pharmaceutic industry. Through interviews applied to the responsible of innovation, we show how had been the development of researchers, government expenditure and the development of science and technology during 2010 to 2015. 


\section{Innovación en las sociedades del conocimiento}

$\mathrm{E}$ n la teoría del desarrollo económico de Schumpeter se considera que los procesos de innovación y desarrollo tecnológico en combinación con factores de producción, recursos naturales y humanos y con aspectos intangibles como el conocimiento, generan un proceso de transformación social y económico (Schumpeter, 1934). En las economías desarrolladas, este proceso de transformación ha impactado también a las universidades, las cuales persiguen un papel más emprendedor, por lo tanto necesitan desarrollar de forma conjunta la investigación publicable y la investigación comercializable (Chang, Yang, Martin, \& Chi, 2016), es decir, aquella que puede ser transferida y generar un desarrollo económico.

Sin embargo, hasta principios de este siglo, en Latinoamérica esta transformación de las universidades aún está por realizarse de manera generalizada, pues aún mantienen un relativo aislamiento respecto a las comunidades que las sostienen (Arocena \& Sutz, 2001), y se debaten entre la posibilidad de contribuir con conocimiento al desarrollo económico o atender lo que consideran su responsabilidad como instituciones públicas: atender la relevancia social de los problemas que intentan resolver (Arocena \& Sutz, 2005).

En este contexto "las sociedades emergentes no pueden contentarse con ser meros componentes de una sociedad mundial de la información y tendrán que ser sociedades en las que se comparta el conocimiento" (UNESCO, 2005, p.5).

De acuerdo a la OCDE "México se ha tardado en darse cuenta de la importancia de la inversión en la innovación como un motor de crecimiento y competitividad" (OECD, 2008, p.7), en tal sentido destaca "la necesidad de crear políticas específicas para fomentar la ciencia, la tecnología y la innovación para mejorar el rendimiento del sistema que permita contribuir más al crecimiento económico" (OECD, 2008, p. 11), pero ¿qué es la innovación y como ocurre en la actualidad? De acuerdo con la OECD, la innovación implica introducir nuevos productos, procesos o métodos considerablemente mejorados, y es el motor de: crecimiento y empleo, mejora de los niveles de vida, aumento de la competitividad, diversificación de la economía y el camino a actividades de valor agregado más alto (OECD, 2010).

En la figura 1, se muestra un esquema que representa un diagrama de flujo y muestra los factores en el proceso de innovación. En el centro de este proceso se encuentra el capital humano que representa la esencia de la innovación al crear, difundir o adaptar tecnologías en el lugar de trabajo. Pero al mismo tiempo este capital humano actúa como consumidor de estas innovaciones, es por eso que se puede observar un bidireccionamiento en esta relación. El capital humano puede jugar un papel de emprendedor, es decir, de aquel participante en el proceso capaz de convertir las ideas en aplicaciones prácticas mediante la creación exitosa de empresas. En este esquema se muestra que las instituciones de educación superior (IES) y los centros de investigación son los encargados de producir y atraer el capital humano necesario para el de desarrollo de innovación. Las IES y los centros de investigación también actúan como puentes indispensables entre los demás participantes, empresas y gobiernos, que finalmente constituyen el triduo involucrado en la producción de innovación en los países (OECD, 2010).

Se puede observar entonces como "la creación, difusión y aplicación del conocimiento son imprescindibles para la habilidad de las empresas y los países para innovar y prosperar en una economía mundial cada vez más competitiva" (OECD, 2010). Es decir, "La ciencia sigue estando en el centro de la innovación" en tal sentido se exigen reformas para mantener la excelencia y aumentar la colaboración con el sector empresarial (OECD, 2010).

Lo anterior empata con la idea de la adopción de nuevas tendencias de producción y difusión del conocimiento hacia el llamado modo 2 de Gibbons, "caracterizado por un alejamiento de la búsqueda de principios fundamentales para avanzar hacia modelos de investigación orientados hacia resultados contextualizados" (Gibbons et al., 1997, p. 33).

En el contexto industrial, el tránsito de lo teórico a lo aplicable, se debe en gran medida a la "densidad de la comunicación" mostrada en los sistemas de ciencia y tecnología en dos niveles:

- Comunicación entre ciencia y sociedad. Las nuevas demandas de responsabilidad y de mayor comunicación entre expertos científicos y el público, que emanan de la difusión de la educación superior a través de la sociedad, y generan nuevas formas de comunicación que vincula diversos tipos de instituciones, dando paso al surgimiento de comunidades híbridas que requieren procesos de transmisión del conocimiento científico y tecnológico a la sociedad (Gibbons et al. 1997).

- Comunicación entre los científicos. Dada por la movilidad de los científicos, que genera en ellos ideas más creativas y novedosas debido al intercambio de experiencias, instrumentos $\mathrm{y}$ principios con otros científicos de diferentes lugares y en segundo lugar con la forma en que establecen prioridades y seleccionan los problemas a abordar (Gibbons et al., 1997).

En el modo 2, el descubrimiento, la aplicación y el uso del conocimiento se encuentren interconectados, a través de un mecanismo de 
expansión del mercado para el conocimiento, en tal sentido las empresas tienden que buscar nuevos tipos de vínculos con las universidades. Para las empresas "las relaciones de colaboración son bienvenidas porque la acumulación de conocimiento específico para la empresa depende de un ambiente más amplio, posiblemente global, en el que se produce el conocimiento"(Gibbons et al., 1997, p. 68).

Esto puede ser visto como el desarrollo de capacidades para la generación de conocimiento que incidan en el desarrollo económico a través de vínculos estrechos entre universidades o centros de investigación, empresas, gobierno y sociedad; lo que engloba el concepto vinculación en términos de lo propuesto por Casalet y Casas que definen vinculación como una relación de intercambio y cooperación entre las instituciones educativas y el sector productivo, que tiene como objetivos promover el avance académico, el desarrollo científico y tecnológico y la solución de problemas planteados por la sociedad (Casalet y Casas, 1998). Es decir construir una mejor sociedad basada en el conocimiento que usa la investigación y habilidades colectivas.

Según Figueroa Sepulveda (2013) las Universidades Públicas en latinoamericana no satisfacen la función de generar "conocimiento para la producción y, si es el caso, el procesamiento de las aplicaciones productivas del mismo" (Martínez de Ita, Piñero, \& Figueroa Delgado, 2013, p.12). Esto no significa que I+D sea una función exclusiva de las universidades, otras organizaciones de la sociedad también cuentan con esta función, por ejemplo los departamentos de investigación y desarrollo de las empresas.

Figura1. Diagrama de producción de innovación

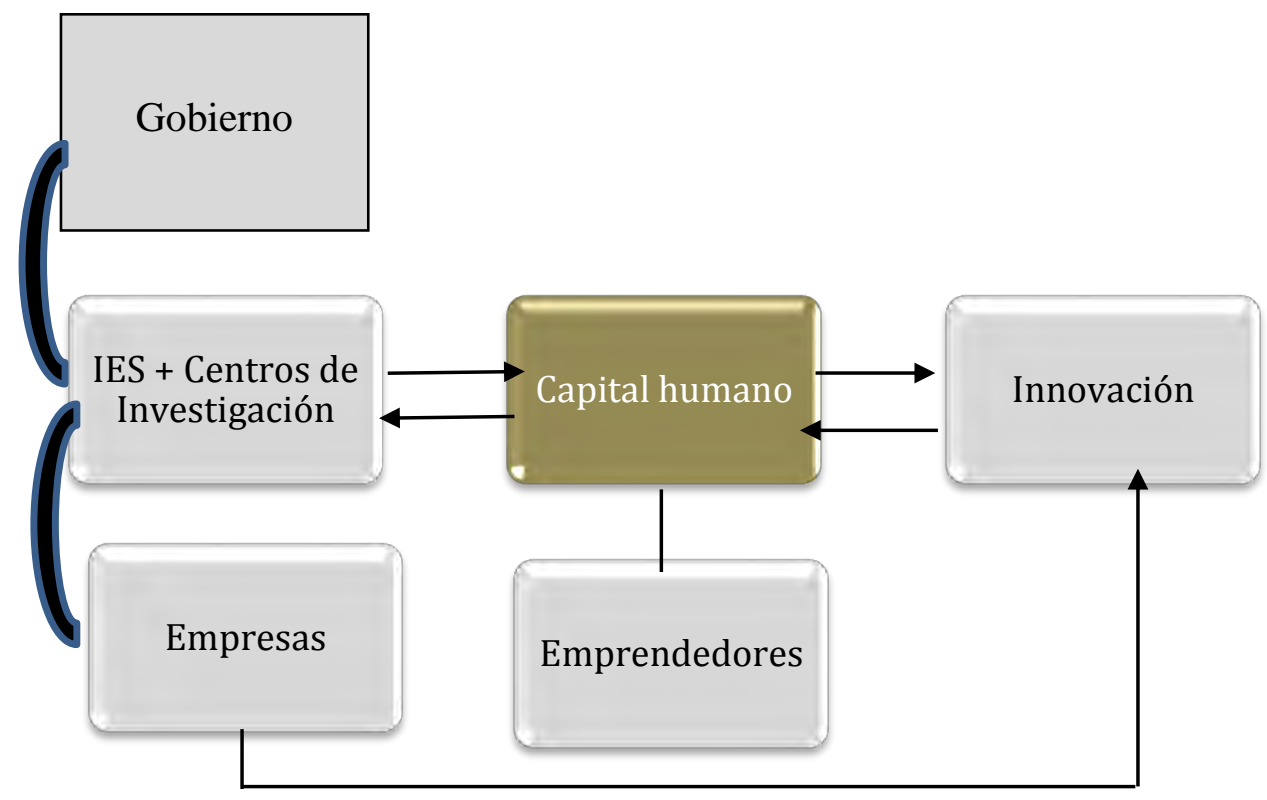

Nota: Adaptación propia del modelo de innovación estratégica descrito por la OECD.

Fuente: OECD. (2010). THE OECD INNOVATION STRATEGY: GETTING A HEAD START ON TOMORROW.

\section{Universidad de Guadalajara y Sector Productivo Farmacéutico ¿Representatividad y extrapolación?}

Saber sí en el estado de Jalisco (México) se ha adoptado a esta nueva forma de producción de conocimiento a través de una vinculación efectiva entre la Universidad de Guadalajara (UdeG) y el sector productivo Farmacéutico, es la tarea que ocupa a este trabajo de investigación.
¿Por qué el Estado de Jalisco, UdeG y el Sector Productivo Farmacéutico?

\subsection{Jalisco}

- Jalisco ocupa el lugar 4 a nivel nacional por su número de habitantes (INEGI, 2010).

- Se encuentra entre las economías más importantes de México con una aportación al Producto Interno Bruto (PIB) nacional que representó el 6.5\% en 2014 (INEGI, 2014) 
- Con una distribución del PIB estatal por sector de actividad encabezada por las actividades terciarias (comercio, servicios y transporte) que representó en 2014 el 63.27\% (INEGI, 2014)

- Además cuenta con un número de investigadores que corresponde al $5.1 \%$ del total nacional distribuido por áreas de la ciencia como se muestra en la figura 2 (Secretaría de Economía, 2016).

Por último el argumento que sostiene la elección de Jalisco se basa en la visión propuesta en la Agenda de innovación para el estado que establece que "en 2030, Jalisco será un estado referente en innovación en la República Mexicana dentro de las industrias vocacionales del estado, ya con una cultura que impulse la creatividad y el emprendimiento de base tecnológica e innovador" (CONACYT, 2015, p.41)

\subsection{Universidad de Guadalajara (UdeG)}

Debido a que la UdeG es "la universidad pública estatal con el mayor número de miembros del SNI y se ubica entre las cuatro primeras instituciones de educación superior en este indicador en el ámbito nacional" (UdeG, 2016)

\subsection{Sector Productivo Farmacéutico}

- Se estima que en 2020, el centro de desarrollo tecnológico de la industria farmacéutica para América Latina estará en México (Secretaría de Economía, s/f). Para ese año, la industria farmacéutica será uno de los pilares del modelo industrial de producción de bienes y servicios de alto valor agregado y productividad en el país (Secretaría de Economía, s/f)

- El sector del comercio en Jalisco es el que tiene el mayor porcentaje de participación en el PIB estatal con un 23.5\%; seguido por la industria manufacturera con un $21.3 \%$ (Secretaría de Economía, s/f), dentro del cual se ubica a la industria farmacéutica.
- Jalisco ocupa el segundo lugar a nivel nacional con mayor actividad económica por parte del sector farmacéutico (el primer lugar lo tiene el Distrito Federal) (Secretaría de Economía, s/f).

- Salud e industria farmacéutica han sido seleccionada como las áreas de especialización en la agenda de Jalisco (CONACYT, 2015), donde "se han identificado 10.034 unidades económicas en el estado según DENUE, 9,905 enfocadas a la prestación de servicios de salud y 129 en el desarrollo de productos farmacéuticos" (CONACYT, 2015, p.62)

- De acuerdo al análisis FODA presentado en la agenda, para fines del propósito de este trabajo, cabe resaltar "la escasa vinculación entre academia y empresa”(CONACYT, 2015, p.63) en este sector, y la "posible centralización de la I+D global del sector en unos pocos destinos que ya lideran el sector tecnológicamente" (CONACYT, 2015, p.63)

- Por lo tanto focalizar este trabajo en el sector farmacéutico podría contribuir a incrementar el tamaño del clúster en Jalisco, favoreciendo el crecimiento económico del estado y el país, mostrar las fortalezas y obstáculos que existen en la vinculación entre universidad y empresa podría ayudar a incrementar las relaciones entre ambos fortaleciendo la producción de conocimiento articulado, que a su vez favorecería la descentralización este sector industrial propiciando el establecimiento de empresas en esta zona del país, las cuales podrían aprovechar el capital humano de la UdeG.

Caracterizar el proceso de vinculación que se lleva a cabo la Universidad de Guadalajara (UdeG) para establecer actividades de trabajo colaborativo con el Sector Productivo Farmacéutico. Con el fin de mostrar que tan alejado o cercano se encuentra el proceso de producción de conocimiento colaborativo tendiente a la innovación.

Figura 2. Número de Investigadores por área de la Ciencia, 2015

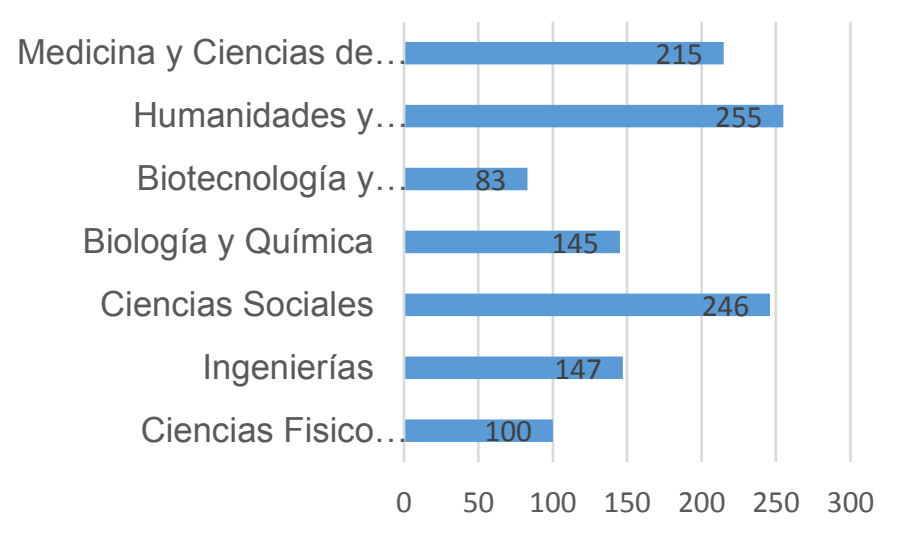

Fuente: Secretaría de Economía. (2016). PROMÉXICO. Inversión y Comercio Jalisco. Recuperado a partir de www.promexico.gob.mx 


\section{Metodología}

Con el fin de conocer las percepciones de los propios actores de la universidad y los requerimientos de la industria farmacéutica para concretar proyectos de vinculación con la UdeG, se diseñó una entrevista no estructurada la cual fue aplicada a dos responsables de investigación y desarrollo de las dos principales empresas de la industria farmacéutica establecidas en Jalisco, de estas entrevistas se rescatan hallazgos tales como variables y experiencias que nos permiten mostrar un panorama de datos reales sobre las practicas que se llevan a cabo en este proceso y mostrar una evaluación del acercamiento al modo de producción de bienes y servicios de valor agregado.

Las empresas seleccionadas como estudio de caso en este trabajo representan el primer y segundo lugar, dentro del estado de Jalisco, en orden de participación en el mercado mexicano de productos OTC (over the counter) o de libre acceso. Seleccionar bajo este criterio es importante ya que el $43 \%$ de las ventas de este tipo de medicamentos (OTC) en el mundo está concentrado en 14 empresas (ProMéxico, 2013).

Las dos empresas visitadas han incluido innovación en diferentes áreas de conocimiento, la cual responde a la necesidad de ser más competitivas. A partir de las áreas en que los responsables de dichas empresas mencionaron que incluyeron innovación y además que buscaron un nexo con la UdeG, se han seleccionado 4 actores pertenecientes a la UdeG para ser entrevistados, los cuales representan cada uno un área de investigación de las mencionadas por las empresas. En la figura 3 se muestra esta línea de relación.

Figura 3. Áreas de colaboración posible entre empresas A y B con la UdeG

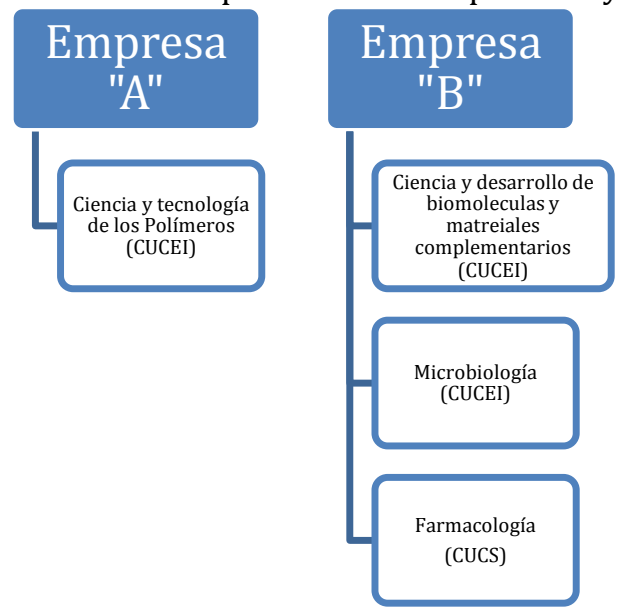

Nota: Elaboración propia.

Mediante las entrevistas realizadas a investigadores de la UdeG se obtienen las variables que permiten mostrar las fortalezas y oportunidades, internas en la universidad. Las fortalezas son las virtudes o capacidades internas que le permiten enfrentar con éxito cambios o retos (Villa Rivera, 2004) y las oportunidades aquellas circunstancias que se espera ocurran en el entorno $\mathrm{y}$ que pueden ser aprovechadas o pueden tener un impacto positivo en las organizaciones(Villa Rivera, 2004).

En esta fase del proceso metodológico se pondrá en análisis algunas de las consideraciones en la forma de producción de conocimiento propuesto por Gibbons en el modo 2, para conocer qué tan cercano se encuentra el binomio UdeG-sector farmacéutico a contribuir con el desarrollo económico de la sociedad.

Con la finalidad de enriquecer el panorama del entorno entre la vinculación UdeG-Industria Farmacéutica y empatar con la caracterización del modo 2 de Gibbons, es necesario realizar un análisis documental acerca de las tendencias que nos permita verificar:

I. El crecimiento de personas calificadas en las áreas de conocimiento mencionadas en la figura 3. Para tal efecto se ha considerado una trayectoria temporal 2010 a 2015, esto se realiza a través de un análisis de tendencia del número de investigadores miembros del Sistema Nacional de Investigadores (SNI) pertenecientes a la UdeG en relación con las áreas de conocimiento: Biología y Química clasificada como área 2 en el CONACYT, 3. Medicina y Ciencias de la Salud, 6. Biotecnología y Ciencias Agropecuarias y 7. Ciencias de la Ingeniería.

II. La inversión gubernamental en el desarrollo de ciencia y tecnología en este ramo, mediante el análisis de financiamiento público obtenido a través del Programa de Estímulos a la Innovación, que es el "programa de apoyo para las empresas que 
invierten en proyectos de investigación, desarrollo de tecnología e innovación dirigidos al desarrollo de nuevos productos, procesos o servicios" (CONACYT, 2014b). Dicho programa comprende 3 modalidades:

INNOVAPYME (Innovación tecnológica para las micro, pequeñas y medianas empresas)

Modalidad dedicada exclusivamente a propuestas y proyectos cuyo proponente sea empresas MIPYMES.

En esta modalidad las empresas podrán presentar propuestas de manera individual o vinculada con IES, CI o ambos (CONACYT, 2014b).

INNOVATEC (Innovación Tecnológica para las grandes empresas)

Modalidad dedicada exclusivamente a propuestas y proyectos cuyo proponente sea empresas grandes.

En esta modalidad las empresas podrán presentar propuestas de manera individual o vinculada con IES, CI o ambos (CONACYT, 2014b).

PROINNOVA (Proyectos en red orientados a la innovación)

Modalidad dedicada exclusivamente a propuestas y proyectos que se presenten en vinculación con al menos dos IES, o dos CI o uno de cada uno (CONACYT, 2014b).
Se presentan los montos de financiamiento otorgados a empresas del ramo farmacéutico establecidas en Jalisco para los años 2009 al 2012, esta temporalidad obedece a la disponibilidad de los datos.

\section{Resultados}

De acuerdo a los datos de la figura 4, se mantiene un número de investigadores casi estable entre 2010 y 2013. En algunos casos como el área de Ciencias de Ingeniería, Biología y Química para 2012 hay una caída en la tendencia de crecimiento; de igual forma sucede en 2011 para Biotecnología y Ciencias Agropecuarias; solo el área de Medicina y Ciencias de la Salud mantiene una tendencia de crecimiento.

Cabe resaltar que para el año 2014, no se reportan datos por parte del SNI.

Como se muestra en la figura 4 de 2013 a 2015 hay un claro crecimiento de personas calificadas con experiencia empírica en todas las áreas determinadas como clave. Las áreas de Biotecnología y Ciencias Agropecuarias y Ciencias de la Ingeniería tuvieron un crecimiento de más del 100\%; el área de Biología y Química de aproximadamente el 50\%; y el área de Medicina y Ciencias de la Salud cerca de 30 puntos porcentuales de crecimiento.

Figura 4. Crecimiento de Investigadores Miembros del SNI 2010-2015 en Áreas Específicas de Conocimiento

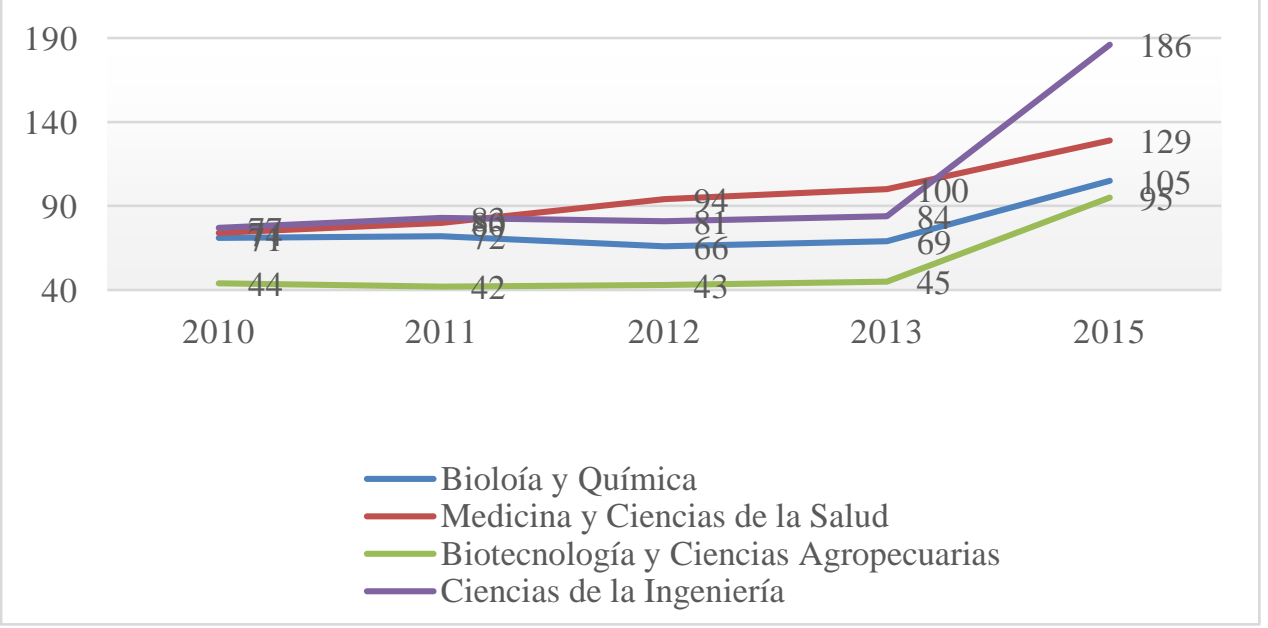

Nota: Elaboración propia. Fuente: CONACYT. (2016). Base de Datos Miembros SNI. Histórico 2015.(CONACYT, 2016).

En la figura 5 se muestra el comportamiento en el otorgamiento de fondos a través de las tres modalidades del Programa de Estímulos a la Innovación, se observa que el programa INNOVAPYME e INNOVATEC son los que han tenido un comportamiento más estable. El programa PROINNOVA refleja un decaimiento considerable entre 2009 y 2011, para 2012 se da un crecimiento que representa cerca del $200 \%$. Se aplica regresión lineal para establecer la tendencia en las tres modalidades, y se observa que la pendiente es negativa en todos los casos, siendo mayor la que corresponde al programa PROINNOVA. 
Figura 5. Monto de estímulo a la innovación otorgado a empresas farmacéuticas en Jalisco con regresión líneal.

\section{Monto de estímulo a la innovación otorgado a empresas farmacéuticas en Jalisco}

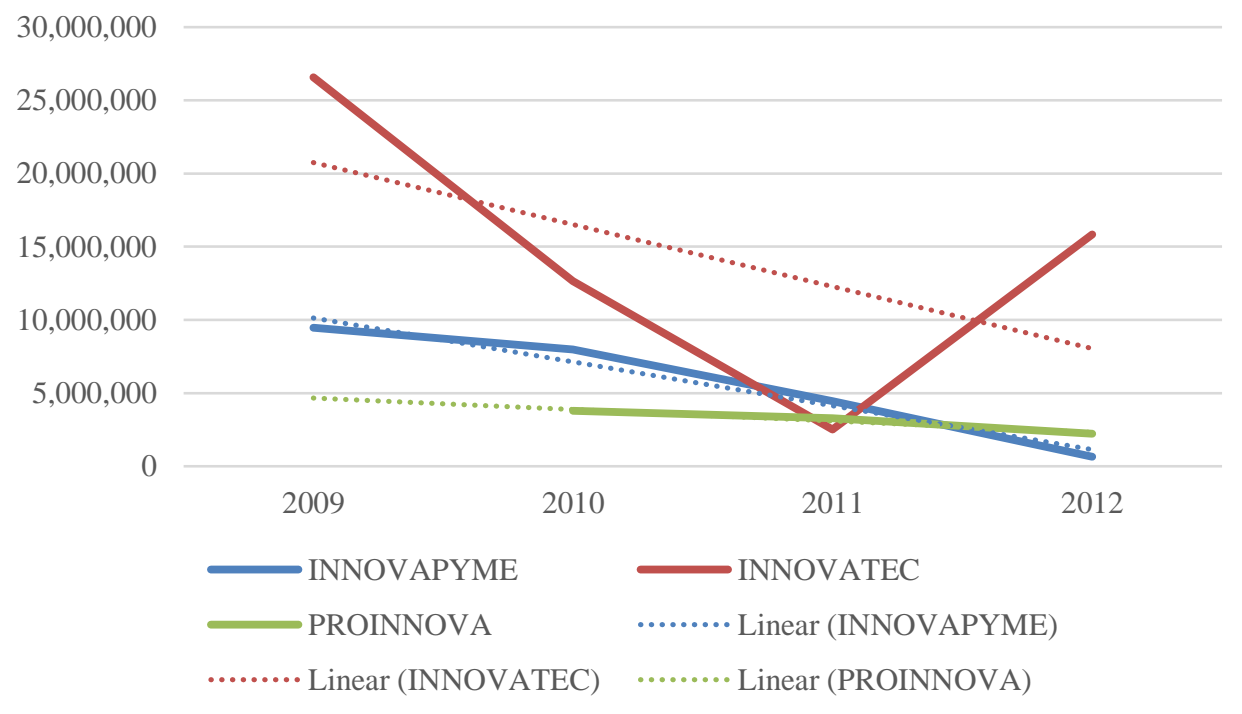

Nota: Elaboración propia.

Fuente: CONACYT. (2014a). Padrón de Beneficiarios 2009-2013 PEI. Recuperado a partir de http://www.conacyt.mx/index.php/fondos-y-apoyos/programa-de-estimulos-a-la-innovacion (CONACYT, 2014a).

En la tabla 1 se muestra una relación de las características que definen la producción del conocimiento hacia un sentido más enfocado a la innovación o al desarrollo de bienes y servicios de Tabla 1. Resultados valor agregado. Los parámetros han sido establecidos en apego al modelo de Gibbons para la nueva producción de conocimiento en sociedades contemporáneas (Gibbons et al., 1997, p.70-81).

\begin{tabular}{|c|c|c|}
\hline Variable & Parámetro & Resultados \\
\hline $\begin{array}{l}\text { Personas } \\
\text { Calificadas }\end{array}$ & $\begin{array}{l}\text { Hay un crecimiento de personas } \\
\text { calificadas en alguna disciplina } \\
\text { científica o técnica con formación en } \\
\text { investigación empírica. }\end{array}$ & $\begin{array}{l}\text { Número de investigadores casi estable entre } 2010 \text { y } 2013 \text {. Se } \\
\text { muestran en la figura } 4 \text {. } \\
\text { Crecimiento de personas calificadas entre } 2013 \text { a } 2015 \text {. Se } \\
\text { muestran en la figura } 4 \text {. }\end{array}$ \\
\hline $\begin{array}{c}\text { Disposición de la } \\
\text { colaboración }\end{array}$ & $\begin{array}{l}\text { Quienes poseen habilidades están } \\
\text { dispuestos a reunirse para la } \\
\text { resolución de problemas difíciles y } \\
\text { desafiantes. }\end{array}$ & $\begin{array}{l}\text { Por una parte existen académicos de la UdeG que manifiestan } \\
\text { un alta disposición para el trabajo colaborativo en virtud de: } \\
\text { - Acercarse a la dinámica de las empresas. } \\
\text { - Demostrar que lo que hacen en el laboratorio de } \\
\text { investigación es aplicable en la industria. } \\
\text { - Involucrar alumnos } \\
\text { Por otra parte algunos de los entrevistados mencionan ya no } \\
\text { querer trabajar con la industria, debido a que este trabajo } \\
\text { colaborativo implica: } \\
\text { - Estar impulsando la "maquinaria universitaria" para } \\
\text { - lograr concretar proyectos. } \\
\text { Invertir demasiado tiempo en múltiples funciones } \\
\text { desde obtener insumos, gestionar recursos, realizar } \\
\text { trámites. } \\
\text { Riesgo de no publicar y por lo tanto salir del SNI. }\end{array}$ \\
\hline $\begin{array}{l}\text { Producción de } \\
\text { conocimiento }\end{array}$ & $\begin{array}{l}\text { Las empresas participan en la } \\
\text { producción del conocimiento, para } \\
\text { poder comercializarlo, por lo tanto } \\
\text { desarrollan nuevos vínculos con las } \\
\text { universidades y los laboratorios } \\
\text { gubernamentales y con otras } \\
\text { empresas. } \\
\text { Tanto el sector académico como el }\end{array}$ & $\begin{array}{l}\text { Una de las empresas entrevistadas menciona que no está } \\
\text { interesada en crear vínculos con la UdeG debido a la falta de } \\
\text { confianza en la institución. } \\
\text { Mientras que la otra detecta gran participación por parte de } \\
\text { los académicos en los proyectos que la empresa ha necesitado, } \\
\text { por lo tanto vincularse con la UdeG en la producción de } \\
\text { conocimiento resulta sumamente fructuoso. } \\
\text { Por su parte los académicos reconocen que: }\end{array}$ \\
\hline
\end{tabular}




\begin{tabular}{|c|c|c|}
\hline Variable & Parámetro & Resultados \\
\hline & $\begin{array}{l}\text { industrial se convierten en } \\
\text { participantes en la producción de un } \\
\text { conocimiento que tenga potencial } \\
\text { para crear riqueza. } \\
\text { La producción del conocimiento se } \\
\text { ve configurada por las necesidades e } \\
\text { intereses de algunos de sus } \\
\text { consumidores potenciales. }\end{array}$ & $\begin{array}{l}\text { - Es muy difícil hacerles creer a las empresas que } \\
\text { pueden resolver el problema, debido a que hay mucha } \\
\text { desconfianza de las personas encargadas de generar } \\
\text { desarrollo en una empresa en la UdeG como } \\
\text { institución. } \\
\text { Falta como credibilidad en la universidad, algunos } \\
\text { derroches de mala publicidad han hecho que el que se } \\
\text { acerque lo haga con cierto recelo. } \\
\text { Desde el interior los investigadores se esfuerzan en demostrar } \\
\text { que ellos hacen las cosas bien, que pueden resolver problemas, } \\
\text { e intentan limpiar lo que está en sus manos de acerca de esa } \\
\text { mala imagen. } \\
\text { En cuanto a la configuración en la producción de conocimiento, } \\
\text { los entrevistados coinciden en que los investigadores se } \\
\text { apegan a los requerimientos que solicita la empresa, } \\
\text { adaptando su metodología a las normas que se requieren a } \\
\text { nivel industrial, }\end{array}$ \\
\hline $\begin{array}{l}\text { Inversión } \\
\text { gubernamental } \\
\text { en ciencia y } \\
\text { tecnología }\end{array}$ & $\begin{array}{l}\text { Las políticas de ciencia y tecnología } \\
\text { ponen mayor énfasis en la } \\
\text { financiación pública para propósitos } \\
\text { económicos. }\end{array}$ & $\begin{array}{l}\text { El Programa de Estímulo a la Innovación enfatiza el } \\
\text { otorgamiento de recursos económicos para ser invertidos en } \\
\text { proyectos que impacten en el desarrollo económico. La } \\
\text { tendencia en el uso de este recurso va a la baja. Se muestran en } \\
\text { la figura } 5 .\end{array}$ \\
\hline $\begin{array}{l}\text { Difusión del } \\
\text { conocimiento }\end{array}$ & $\begin{array}{l}\text { La tarea de las unidades de } \\
\text { investigación y de las universidades } \\
\text { consiste en trasladar el } \\
\text { conocimiento a través de fronteras } \\
\text { institucionales. } \\
\text { Aparece la necesidad de fortalecer la } \\
\text { red de distribución y flujo del } \\
\text { conocimiento desde las } \\
\text { universidades y el gobierno hacia los } \\
\text { centros de riqueza. } \\
\text { La distribución de los resultados de } \\
\text { investigación de los laboratorios } \\
\text { gubernamentales o de las } \\
\text { universidades va más allá de su } \\
\text { comunidad de especialistas a cuyo } \\
\text { servicio se han creado. }\end{array}$ & $\begin{array}{l}\text { En la UdeG se cuenta con una oficina de vinculación } \\
\text { lamentablemente coinciden académicos y empresarios en que: } \\
\text { - No se otorga formalmente información sobre servicios } \\
\text { - } \quad \text { que la UdeG ofrece en materia de vinculación. } \\
\text { - } \quad \text { Falta mayor promoción. } \\
\text { - La oficina de vinculación no sabe de lo que son capaces } \\
\text { - } \quad \text { Los investigadores. } \\
\text { cuentan. } \\
\text { El acercamiento obedece al conocimiento de la UdeG debido } \\
\text { nexos personales, no a una buena difusión de conocimiento, } \\
\text { producto de una distribución de investigaciones. }\end{array}$ \\
\hline
\end{tabular}

Nota: Elaboración Propia.

\section{Conclusiones}

Pocos académicos con aptitudes para la investigación desarrollo e innovación tienen la voluntad de trabajar de forma colaborativa, solo este reducido porcentaje ha detectado en la vinculación o transferencia de conocimiento una situación conveniente. Al hablar de voluntad, es interesante resaltar que aquellos casos exitosos que fueron compartidos en la elaboración de este trabajo, obedecen al comportamiento, perspicacia, actitud, aptitud y perseverancia de individuos particulares.

De acuerdo a los hallazgos detectados en este documento la mayoría de los académicos están cansados de direccionar sus fuerzas para librar batallas ante la burocracia institucional, con el fin de mejorar la respuesta en tiempo y procesos legales ante la industria. Lo anterior representa el riesgo de perder beneficios formales y establecidos, lo que permite constatar la incapacidad institucional "para incentivar entre investigadores y estudiantes el contacto con empresas y el trabajo de investigación aplicada, e incluso de aplicación industrial" (Arechavala Vargas, 2013).

La producción del conocimiento entre la UdeG y el sector productivo farmacéutico tiene vínculos endebles que se deben a juicios de valor producto de conocimiento de malas prácticas dentro de la universidad. Los investigadores miembros de la institución muestran una voluntad férrea para fortalecer estos vínculos. Tal como propone la UNESCO, uno de los caminos para crear y restaurar la confianza proviene de la transparencia en los flujos de información (UNESCO, 2005). Es por esta razón que resulta apremiante tomar acciones que permitan acrecentar los nexos entre sector productivo y universidad.

La actuación gubernamental deja en evidencia políticas que buscan un acrecentamiento en el desarrollo económico a través de la otorgación de recursos concursables, pero el efecto de estas políticas de fomento ha ido en decaimiento. 
Vinculación en materia de innovación entre la Universidad de Guadalajara...

Como sugiere la UNESCO, es necesario que se supere la brecha científica para lograr el tránsito hacia sociedades del conocimiento (UNESCO, 2005). El desafío consiste en encaminar el desarrollo científico a los procesos productivos de forma interrelacionada, superando los factores endógenos presentes.
Finalmente en lo que refiere a la difusión de conocimiento se confirma una incapacidad por parte de la UdeG para "movilizar el conocimiento en labores de apoyo a la industria" (Arechavala Vargas, 2013). 


\section{Referencias}

Arechavala Vargas, R. (2013). Las universidades públicas mexicanas: los retos de las transformaciones institucionales hacia la investigación y la transferencia de conocimiento. Presentado en XV Latin IberoAmerican Conference on Management of Technology.

Arocena, R., \& Sutz, J. (2001). Changing knowledge production and Latin American universities. Research Policy, 30(8), 1221-1234. http://doi.org/10.1016/S0048-7333(00)00143-8

- (2005). Latin American Universities: From an Original Revolution to an Uncertain Transition. Higher Education, 50(4), 573-592.

Casalet, M., \& Casas, R. (1998, septiembre). Un diagnóstico sobre la vinculación Universidad-Empresa CONACyTANUIES. Revista de la Educación Superior, 27(107), 1-5.

Chang, Y.-C., Yang, P. Y., Martin, B., \& Chi, H.-R. (2016). Entrepreneurial universities and research ambidexterity: Amultilevel analysis. Elsevier.

CONACYT. (2014a). Padron de Beneficiarios 2009-2013 PEI. Recuperado a partir de http://www.conacyt.mx/index.php/fondos-y-apoyos/programa-de-estimulos-a-la-innovacion

- (2014b). Programa de Estímulos a la Innovación. Recuperado el 5 de febrero de 2016, a partir de http://www.conacyt.mx/index.php/fondos-y-apoyos/programa-de-estimulos-a-la-innovacion

- (2015). Agenda de Innovación de Jalisco. Recuperado a partir de www.agendasinnovacion.mx

- (2016). Base de Datos Miembros SNI. Historico 2015.

Gibbons, M., Limoges, C., Nowotny, H., Schwartzman, S., Scott, P., \& Trow, M. (1997). La nueva producción del conocimiento. La dinámica de la ciencia y la investigación en las sociedades contemporaneas (1a ed.). Barcelona: Ediciones Pomares-Corredor.

INEGI. (2010). Población, Hogares y Vivienda. Recuperado a partir de http://www3.inegi.org.mx/

- (2014). Censos Económicos. Recuperado a partir de http://www3.inegi.org.mx/

Martínez de Ita, M. E., Piñero, F. J., \& Figueroa Delgado, S. A. (2013). El papel de la universidad en el desarrollo (1a ed.). México.

OECD. (2008). OECD Reviews of Innovation Policy MEXICO Overall Assessment and Recommendations.

- (2010). THE OECD INNOVATION STRATEGY: GETTING A HEAD START ON TOMORROW.

ProMéxico. (2013). Industria Farmacéutica. Unidad de Inteligencia de Negocios. Recuperado a partir de http://mim.promexico.gob.mx/work/sites/mim/resources/LocalContent/368/2/130820_DS_Farmaceu tica_ESP.pdf

Schumpeter, J. (1934). The theory of economic development (1a ed.). Estados Unidos de América: Harvard economics studies.

Secretaría de Economía. (2016). PROMÉXICO. Inversión y Comercio Jalisco. Recuperado a partir de www.promexico.gob.mx

- (s/f). El sector farmacéutico en México. Recuperado a partir de embamex.sre.gob.mx

UdeG. (2016). Inicia UdeG 2016 con 927 especialistas en el SNI [Noticia]. Recuperado a partir de http://www.udg.mx/

UNESCO. (2005). Hacia las sociedades del conocimiento.

Villa Rivera, E. (2004). Construir el futuro en el presente. Elementos conceptuales y metodológicos para la planeación y desarrollo de instituciones de educación Superior. Materiales para la Reforma No. 16. IPN. 